\title{
Modified Charlson comorbidity index as a pre-operative selection tool for liver transplantation in elderly patients
}

\author{
YoungRok $\mathrm{CHOI}^{* 1,2}$, Jiho $\mathrm{CHOl}^{2}$, Su Young HONG ${ }^{1,2}$, Sanggyun $\mathrm{SUH}^{1,2}$, Kwangpyo HONG ${ }^{1,2}$, Eui Soo HAN ${ }^{1,2}$, \\ Jeong-Moo LEE ${ }^{1,2}$, Suk Kyun HONG ${ }^{1,2}$, Nam-Joon $\mathrm{Yl}^{1,2}$, Kwang-Woong LEE ${ }^{1,2}$, Kyung-Suk SUH ${ }^{1,2}$ \\ Department of Surgery, 'Seoul National University Hospital, ${ }^{2}$ Seoul National University College of Medicine, Seoul, Korea
}

Introduction: Liver transplantation is an effective treatment for end-stage liver disease and hepatocellular carcinoma. The number of elderly patients who undergo liver transplantation is increasing. Although it has been known that the overall outcome in elderly patients is no different from the younger ones, there is no tool to predict the prognosis of elderly patients. Therefore, we hypothesized that modified Charlson comorbidity index (CCI), which includes 5 comorbidities, can predict the outcome of elderly patients.

Methods: We conducted a retrospective study of 155 patients over 65 years old who underwent liver transplantation at Seoul National University Hospital from August 2011 to May 2019. Modified CCI score (MODIFIED CCI) including coronary disease, Chronic Obstructive Pulmonary Disease, Diabetes Mellitus, connective tissue disease, and renal insufficiency was calculated. Then, recipients were subcategorized into 2 groups; low MODIFIED CCI group (0-1), and high MODIFIED CCI group (2-5). Proportional hazards analysis was performed to determine the independent effect of each variable on post-transplantation survival using MODIFIED CCI, age at transplantation, gender, Child-Turcotte-Pugh score, Model for End-Stage Liver Disease (MELD) score, and 5-Factor Modified Frailty Index.

Results: There were 41 patients in the high MODIFIED CCI group. The high MODIFIED CCI group showed significantly lower 1-, 3month, and 1, 3, 5-year survival than the low MODIFIED CCI group. In the Cox regression, the MODIFIED CCI $(\mathrm{HR}=2.409)$, gender (HR $=0.447)$, and MELD score $(\mathrm{HR}=1.107)$ remained

Conclusions: Survival after LT is diminished in patients with preoperative high MELD and MODIFIED CCI score $>1$. The MODIFIED CCI can be used. 